\title{
Panels of Immunohistochemical Markers Help Determine Primary Sites of Metastatic Adenocarcinoma
}

\author{
Seog-Yun Park, MD; Baek-Hee Kim, MD; Jung-Ho Kim, MD; Sun Lee, MD; Gyeong Hoon Kang, MD
}

- Context.-Although identification of the primary tumor in patients with metastatic adenocarcinoma has a profound clinical impact, diagnosing the organ of origin is frequently difficult. Because none of the individual immunohistochemical markers used for tissue identification are both site specific and site sensitive, multiple markers are needed to improve the prediction of primary sites.

Objective.-To develop an effective approach to immunohistochemically evaluate metastatic adenocarcinoma for the assignment of a likely primary site of origin.

Design.-Expression profiles of CDX2, cytokeratin (CK) 7, CK20, thyroid transcription factor 1 (TTF-1), carcinoembryonic antigen (CEA), MUC2, MUC5AC, SMAD4, estrogen receptor (ER), and gross cystic disease fluid protein 15 (GCDFP-15) were generated in adenocarcinomas from 7 primary sites, followed by construction of a decision tree and design of multiple-marker panels. Expression of these markers was evaluated immunohistochemically in 314 primary adenocarcinomas (50 cases each of colorectal, gastric, lung, pancreatic, bile duct, and breast, and 14 cases of ovarian origin) using the tissue array method. Results

$\mathbf{M}^{\mathrm{e}}$ etastatic cancer with an unknown primary site accounts for approximately $3 \%$ to $5 \%$ of all malignant neoplasms and, as such, is one of the 10 most frequent cancer diagnoses in humans. ${ }^{1}$ Although modern imaging technology has resulted in improvements in the identification of primary tumors, in most patients the identification remains unknown. ${ }^{2}$ In patients with a metastasis of unknown origin, the primary site of tumor origin is important for management and prognosis. A more precise diagnosis leads to more effective treatment, substantially improving the overall outcome. Therefore, the pathologist has acquired an increasingly important diagnostic role in characterizing the site of origin of these tumors.

The most frequently detected primaries are adenocarcinomas (up to $60 \%$ of all metastatic neoplasms of un-

Accepted for publication May 3, 2007.

From the Department of Pathology, Seoul National University College of Medicine and Cancer Research Institute, Seoul, Korea (Drs Park, B.-H. Kim, J.-H. Kim, and Kang); and the Department of Pathology, Kyung Hee University College of Medicine, Seoul, Korea (Dr Lee).

The authors have no relevant financial interest in the products or companies described in this article.

Reprints: Gyeong Hoon Kang, MD, Department of Pathology, Seoul National University College of Medicine, 28 Yongon-dong, Chongnogu, Seoul 110-744, South Korea (e-mail: ghkang@snu.ac.kr). were validated using 60 cases of metastatic adenocarcinoma with known primaries.

Results.-Organ-specific immunostaining profiles using multiple markers provided high sensitivity, specificity, and positive predictive value in detecting primary adenocarcinomas, as follows: colorectal, TTF-1-/CDX2+/CK7- ${ }^{-} / \mathrm{CK}^{-} \mathrm{C}^{+}$ or $\mathrm{TTF}-1^{-} / \mathrm{CDX2}^{+} / \mathrm{CK}^{-} / \mathrm{CK20}^{-} /\left(\mathrm{CEA}^{+}\right.$or $\left.\mathrm{MUC2}^{+}\right)$; ovarian, $\mathrm{CK}^{+} / \mathrm{MUC}^{+} \mathrm{AC}^{+} / \mathrm{TTF}_{-1}-/ \mathrm{CDX}^{-} / \mathrm{CEA}^{-} / \mathrm{GCDFP}_{-15}{ }^{-}$; breast, GCDFP-15 ${ }^{+} / \mathrm{TTF}^{-1}{ }^{-} / \mathrm{CDX} 2^{-} / \mathrm{CK} 7^{+} / \mathrm{CK20}^{-}$or $\mathrm{ER}^{+} /$ TTF-1 ${ }^{-} / \mathrm{CDX}^{-} / \mathrm{CK}^{-}{ }^{-} / \mathrm{CEA}^{-} / \mathrm{MUC5AC}^{-}$; lung, TTF-1 ${ }^{+}$or TTF-1 $-/$ CDX $^{-} / \mathrm{CK}^{+} / \mathrm{CK}^{-} \mathbf{2}^{-} / \mathrm{GCDFP}^{-15^{-} / \mathrm{ER}^{-} / \mathrm{CEA}^{-} /}$ MUC5AC ${ }^{-}$; pancreaticobiliary, TTF-1 $-/ \mathrm{CDX}^{-} / \mathrm{CK} 7^{+/}$ $\mathrm{CEA}^{+} / \mathrm{MUC} \mathrm{AC}^{+}$; and stomach, TTF-1-/CDX2+/CK7+/ $\mathrm{CK20}^{-}$. Overall, these combined phenotypes correctly predicted the tester samples (metastatic adenocarcinomas with known primaries) in $75 \%$ of cases.

Conclusions.-Determination of tissue-specific immunostaining profiles is valuable in the diagnostic differentiation of metastatic adenocarcinomas from seven common primary sites and should help to correctly predict the organ of primary tumor origin.

(Arch Pathol Lab Med. 2007;131:1561-1567)

known primary origin), for which the seven most common primary sites are colon, breast, ovary, lung, stomach, pancreas, and bile duct. ${ }^{3,4}$ It can be difficult to determine the tissue origin of metastatic adenocarcinoma, however, because its microscopic appearance is not distinctive enough to diagnose by histology alone. By using immunohistochemistry, we can improve identification of the exact origin of metastatic adenocarcinoma. Several immunohistochemical markers have been beneficial for differentiating primary tumors. CDX2 and thyroid transcription factor 1 (TTF-1) are considered sensitive markers for colorectal and pulmonary origins of adenocarcinoma, respectively. ${ }^{5-10}$ The combined expression of cytokeratin (CK) 7 and CK20 is also useful for the diagnosis of the various origins of metastatic adenocarcinoma. ${ }^{11-13}$ Although these immunohistochemical markers assist in narrowing the differential diagnosis of the primary tumor, no single highly sensitive and specific marker exists for each of these 7 sites. Because most adenocarcinoma antigens are not specific for a single tissue type, it seems impossible to develop highly sensitive and specific single markers for each primary adenocarcinoma. For this reason, use of multiple immunohistochemical markers is needed to improve the diagnostic accuracy of primary site prediction.

In the present study, we generated expression profiles 


\begin{tabular}{|c|c|c|c|c|c|}
\hline Antibody to & Clone & Sourcet & Dilution & Retrieval Method & Buffer Solution \\
\hline CEA & II-7 & Dako & $1: 100$ & Microwave, $5 \min \times 3$ & $0.01 \mathrm{M}$ citrate \\
\hline TTF-1 & $8 G 7 G 3 / 11$ & Dako & $1: 100$ & Pressure, $120^{\circ} \mathrm{C}, 3 \mathrm{~min}$ & $0.01 \mathrm{M}$ citrate \\
\hline CDX2 & CDX2-88 & BioGenex & $1: 100$ & Microwave, $5 \mathrm{~min} \times 3$ & $0.01 \mathrm{M}$ citrate \\
\hline CK20 & Ks 20.8 & Dako & $1: 100$ & Microwave, $5 \min \times 3$ & $0.01 \mathrm{M}$ citrate \\
\hline CK7 & OV-TL 12/30 & Dako & $1: 100$ & Microwave, $5 \min \times 3$ & $0.01 \mathrm{M}$ citrate \\
\hline MUC2 & Сср58 & Novocastra & $1: 50$ & Microwave, $5 \min \times 3$ & $0.01 \mathrm{M}$ citrate \\
\hline MUC5AC & $\mathrm{CLH} 2$ & Novocastra & $1: 100$ & Microwave, $5 \min \times 3$ & $0.01 \mathrm{M}$ citrate \\
\hline SMAD4 & B-8 & Santa Cruz Biotechnology & $1: 100$ & Microwave, $5 \min \times 3$ & $0.01 \mathrm{M}$ citrate \\
\hline ER & 1D5 & Dako & $1: 100$ & Microwave, $5 \min \times 3$ & EDTA \\
\hline GCDFP-15 & $23 \mathrm{~A} 3$ & Novocastra & $1: 50$ & Microwave, $5 \min \times 3$ & $0.01 \mathrm{M}$ citrate \\
\hline
\end{tabular}

* All antibodies were mouse monoclonals. The avidin-biotin complex method was used to detect immunostaining in each case. CEA indicates carcinoembryonic antigen; TTF-1, thyroid transcription factor 1; CK, cytokeratin; ER, estrogen receptor; and GCDFP-15, gross cystic disease fluid protein 15.

† Dako, Glostrup, Denmark; BioGenex, San Ramon, Calif; Novocastra, New Castle upon Tyne, United Kingdom; and Santa Cruz Biotechnology, Santa Cruz, Calif.

of 10 immunohistochemical markers in 314 primary adenocarcinomas from 7 main sites, and then selected 7 markers to construct a decision tree and develop panels of multiple markers for each primary site. The panels of multiple markers were superior to single markers in sensitivity, specificity, and positive predictive value (PPV) for primary site diagnosis. The utility of the decision tree and marker panels was validated in an independent tester set of 60 cases of metastatic adenocarcinoma for which the primary site was known.

\section{MATERIALS AND METHODS Patients}

The surgical pathology files of the Department of Pathology, Seoul National University Hospital (Seoul, Korea) were searched for available cases of primary and metastatic adenocarcinoma. A total of 314 primary adenocarcinomas from seven main sites (50 cases each of colorectal, gastric, pulmonary, pancreatic, breast, and bile duct origin, and 14 cases of ovarian cancer) were investigated, and 60 samples from a variety of metastatic sites (lung, liver, brain, soft tissue) were also evaluated for carcinoembryonic antigen (CEA), thyroid transcription factor 1 (TTF-1), CDX2, CK20, CK7, MUC2, MUC5AC, SMAD4, estrogen receptor (ER), and gross cystic disease fluid protein 15 (GCDFP-15) staining. In the group of metastatic adenocarcinomas, the primary sites were as follows: lung $(\mathrm{n}=14)$, breast $(\mathrm{n}=8)$, colon $(\mathrm{n}=14)$, ovary $(\mathrm{n}=4)$, stomach $(\mathrm{n}=10)$, pancreas, and bile duct origin $(\mathrm{n}=$ 10). The antibodies were selected because of their known reactivity with adenocarcinomas of colon, stomach, lung, pancreas, bile duct, breast, and ovary. To select candidate markers, we reviewed Seoul National University Hospital's immunohistochemistry database results for adenocarcinomas of 7 organs for the year 2003. All of the markers we have chosen have a certain selective reactivity for these adenocarcinomas. Furthermore, these antibodies were suitable for formalin-fixed, paraffin-embedded tissue. The tumors of each organ were of the same differentiation grades (moderately differentiated) and include the same histologic types. The set of breast carcinomas comprised all ductal carcinomas, and the group of ovarian carcinomas comprised only the mucinous type of moderately differentiated. In cases of the lung, all of these were adenocarcinomas that were moderately differentiated with acinar pattern. The criterion of histology and differentiation grade of samples we had chosen were decided by the frequency of presentation with metastatic desease. Histologic slides were reviewed by one of the authors, and clinical data with radiologic findings were obtained from medical records. Each type of tissue was identified on the basis of clinical and radiologic findings.

\section{Immunohistochemistry}

Core tissue biopsies ( $2 \mathrm{~mm}$ in diameter) were taken from individual paraffin-embedded adenocarcinomas and were ar- ranged in a new recipient paraffin block (tissue array block) using a trephine apparatus (Superbiochips Laboratories, Seoul, Korea). Each tissue array block contained up to 60 cases, with a total of 8 blocks of tissue array. Using a microtome, tissue array blocks were cut into $4-\mu \mathrm{m}$ slices for immunohistochemical staining. A summary of the antibodies used in this study is shown in Table 1. Immunohistochemical staining was performed using a streptavidin peroxidase procedure (labeled streptavidin-biotin) after an antigen retrieval process using a microwave or a pressure cooker. The staining was carried out in a DAKO Autostainer automatic staining system (Glostrup, Denmark). The slides then were incubated for 15 minutes at $37^{\circ} \mathrm{C}$ in a solution containing $3 \%$ hydrogen peroxide and 3,3'-diaminobenzidine. Neither chromogen enhancer nor endogeneous biotin was used. Positive controls included nonneoplastic bile duct, breast, small intestine, thyroid, gastric glandular epithelium, and apocrine epithelium for CK7, ER, MUC2, TTF-1, MUC5AC, and GCDFP-15, respectively. A section of colonic mucosa was used as a positive control for CK20, CDX2, and SMAD4. Negative controls using mouse immunoglobulin $\mathrm{G}$ at comparable concentrations in place of the primary antibody were included.

\section{Immunohistochemical Evaluation}

The stained slides were assessed without knowledge of clinical or pathologic data. Cases were scored by two of us (S.-Y.P. and B.-H.K.) with respect to the percentage of positive cells and intensity of staining. All disagreements were resolved by evaluation of the slide at a double-headed microscope. All immunohistochemical markers in the study were scored as follows: 0 (no positivity or only very occasional cell staining); $1+(<10 \%$ of cells stained); $2+(10 \%-50 \%$ of cells stained); and $3+(>50 \%$ of cells stained). The intensity of staining was also scored on a categorical scale from 0 to 3: 0 indicated absent; $1+$, very weak, dubious staining; $2+$, definite, mild, or moderate staining; $3+$, definite, strong staining. Only tumor cells stained in the appropriate nuclear or cytoplasmic location were scored. Nuclear staining for TTF-1, CDX2, and ER and cytoplasmic staining with other markers CEA, CK7, CK20, GCDFP-15, MUC2, and MUC5AC were considered positive. For SMAD4, negative labeling was defined as absence of both the cytoplasmic and nuclear compartments. For statistical analyses, only those cases with more than $10 \%(2+$ or $3+)$ of tumor cells exhibiting definite $(2+$ or $3+)$ staining were considered truly positive. Representative images of positive staining are illustrated (Figure 1). Tumors that failed to stain with any marker were excluded.

\section{RESULTS}

\section{Immunohistochemical Profiles of Primary Adenocarcinomas}

The immunohistochemical staining profiles of the primary adenocarcinomas are presented in Table 2. CDX2 

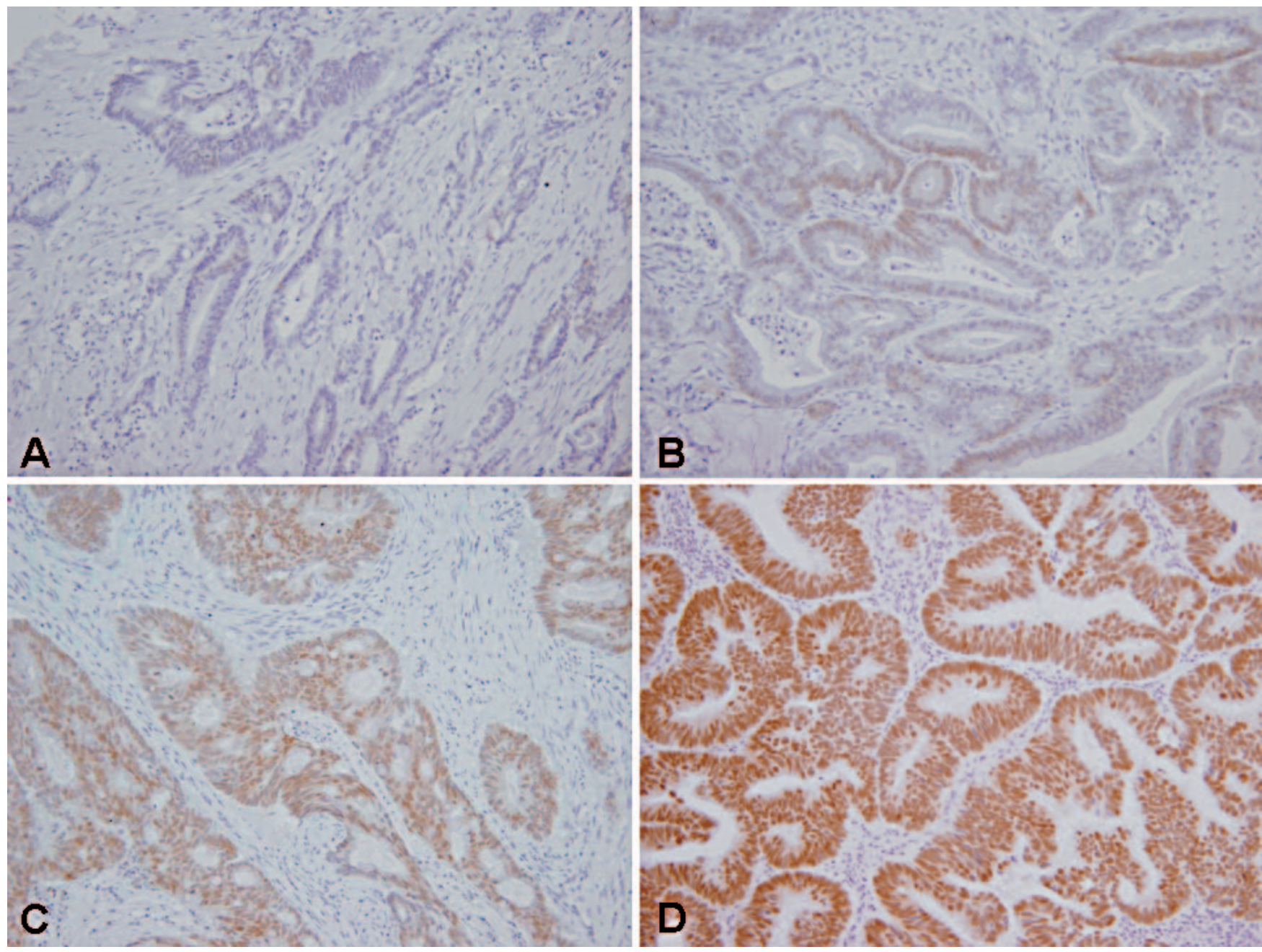

Figure 1. CDX2 immunohistochemical staining of colon cancer. For evaluation purposes and for presentation in the text, we defined the intensity of staining as 0 if staining was absent (A), $1+$ if staining was very weak (B), $2+$ if staining was definite mild to moderate $(C)$, and $3+$ if the staining was strong (D) (immunoperoxidase, original magnifications $\times 200$ ).

was expressed in 46 cases $(93.9 \%)$ of colorectal cancer, 28 cases $(60.9 \%)$ of gastric cancer, and 6 cases $(13 \%)$ of bile duct cancer. None of the pulmonary, ovarian, breast, and pancreatic cases were positive for CDX2. Of the 50 primary pulmonary adenocarcinomas, $44(88 \%)$ had a high level of nuclear staining for TTF-1, whereas none of the 246 adenocarcinomas of nonpulmonary origin showed nuclear staining. A total of $35 \%$ of primary breast cancers expressed GCDFP-15 in this study. In contrast, none of the other primary tissues was positive, except one ( $2 \%)$ colorectal adenocarcinoma. Altogether, with the exception of TTF-1 and GCDFP-15, no single immunohistochemical marker was entirely site specific. Moreover, despite the high specificities of GCDFP-15, this marker did not stain a large proportion of breast cancers, indicating that it is not suitable as a single marker of breast tumors.

\section{Decision Tree Approach}

We also constructed a decision tree (Figure 2) using the ranked specificities and sensitivities of the markers. The most specific and sensitive marker is at the top of the tree, and the first step consists of evaluating TTF-1. If the tumor is TTF-1 positive, then it is from the lung. If negative, we move to the second step, in which GCDFP-15 positivity or negativity is taken into account, leading to the next step, and so on. Using this approach, $65.3 \%$ of the primary sites were predicted correctly. We also found that tumors of pancreatic and bile duct origin mostly expressed the same markers (ie, had similar immunoprofiles and were thus subsequently grouped together).

\section{Approach Using a Panel of Immunohistochemical Markers}

In the current study, we observed that neither single markers nor a decision tree was perfect in determining primary sites. Therefore, we evaluated multiple immunohistochemical markers for each tumor to determine which combination of the 10 markers best predicts primary sites. We evaluated the sensitivities of each marker for each primary site, then selected those markers whose sensitivity was either very high or close to zero. These markers were chosen as the most informative for identification of each primary. For example, the fact that non-breast origin adenocarcinomas almost never express GCDFP-15 supported its inclusion into the panel for discrimination of metastatic adenocarcinomas originating from breast tissue. Table 3 lists the sensitivity and specificity of various combinations of markers for differential diagnosis. The TTF-1 ${ }^{-} / \mathrm{CDX}^{+} /$ 
Table 2. Sensitivities, Specificities, and Positive Predictive Values (PPVs) of Each Immunomarker for 7 Different Tissue Types of Adenocarcinoma*

\begin{tabular}{|c|c|c|c|c|c|c|c|c|c|}
\hline & \multicolumn{3}{|c|}{ Stomach } & \multicolumn{3}{|c|}{ Colon } & \multicolumn{3}{|c|}{ Lung } \\
\hline & $\begin{array}{c}\text { Sensitivity, } \\
\%\end{array}$ & $\begin{array}{c}\text { Specificity, } \\
\%\end{array}$ & $\begin{array}{c}\text { PPV, } \\
\%\end{array}$ & $\begin{array}{c}\text { Sensitivity, } \\
\%\end{array}$ & $\begin{array}{c}\text { Specificity, } \\
\%\end{array}$ & $\begin{array}{c}\text { PPV, } \\
\%\end{array}$ & $\begin{array}{c}\text { Sensitivity, } \\
\%\end{array}$ & $\begin{array}{c}\text { Specificity, } \\
\%\end{array}$ & $\begin{array}{c}\text { PPV, } \\
\%\end{array}$ \\
\hline CEA & 43.5 & 53.2 & 14.6 & 81.6 & 60.7 & 29.2 & 48 & 54.1 & 17.5 \\
\hline TTF-1 & 0 & 82.4 & 0 & 0 & 82.2 & 0 & 88 & 100 & 100 \\
\hline CDX2 & 60.9 & 79.2 & 35 & 93.9 & 86.2 & 57.5 & 0 & 67.5 & 0 \\
\hline CK20 & 23.9 & 72.4 & 13.8 & 87.8 & 85 & 53.8 & 8 & 69.1 & 5 \\
\hline CK7 & 80.4 & 24.4 & 16.4 & 14.3 & 11.3 & 3.1 & 100 & 28.5 & 22.1 \\
\hline MUC2 & 26.1 & 91.2 & 35.3 & 38.3 & 93.9 & 55.9 & 0 & 86.2 & 0 \\
\hline MUC5AC & 50 & 76 & 27.7 & 6.1 & 67.6 & 3.6 & 10 & 68.3 & 6 \\
\hline SMAD4 & 80.4 & 59.2 & 26.6 & 34 & 50.2 & 12.1 & 62 & 55.7 & 22.1 \\
\hline ER & 0 & 85.2 & 0 & 0 & 85 & 0 & 6 & 86.2 & 8.1 \\
\hline GCDFP-15 & 0 & 92.8 & 0 & 6.1 & 93.9 & 16.7 & 0 & 92.7 & 0 \\
\hline
\end{tabular}

* CEA indicates carcinoembryonic antigen; TTF-1, thyroid transcription factor 1; CK, cytokeratin; ER, estrogen receptor; and GCDFP-15, gross cystic disease fluid protein 15.

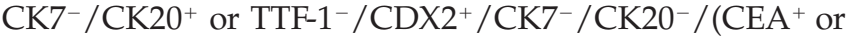
$\mathrm{MUC}^{+}$) phenotype proved to be more specific in detecting colorectal primary tumor localization (sensitivity, 83.7\%; specificity, 97.1\%; PPV, 85.4\%) than was the expression of CDX2 alone (sensitivity, 93.9\%; specificity, $86.2 \%$; PPV, 57.5\%). The combination with the highest sensitivity and specificity for ovarian mucinous adenocarcinomas was positive staining with CK7 and MUC5AC markers and negative staining with TTF-1, CDX2, CEA, and GCDFP-15 (CK7+/MUC5AC ${ }^{+} / \mathrm{TTF}^{+} 1^{-} / \mathrm{CDX}^{-}-/$ $\mathrm{CEA}^{-} / \mathrm{GCDFP}^{-15^{-}}$). The 8-marker combination with the highest sensitivity (94\%) for recognizing adenocarcinomas of lung was positive staining for TTF-1 or an expression pattern of TTF-1-/CDX2-/CK7+/CK20-/GCDFP-15 ${ }^{-}$/ $\mathrm{ER}^{-} / \mathrm{CEA}^{-} / \mathrm{MUC}^{-} \mathrm{AC}^{-}$. Finally, with these various marker panels, the most predictive multiple-marker phenotypes, as determined by a combination of specificity and predictive positive value, were $\mathrm{TTF}-1^{-} / \mathrm{CDX} 2^{-} / \mathrm{CK} 7^{+} / \mathrm{CEA}^{+} /$ MUC5 $\mathrm{AC}^{+}$for pancreatic and bile duct tumors and TTF$1^{-} / \mathrm{CDX} 2^{+} / \mathrm{CK} 7^{+} / \mathrm{CK} 20^{-}$for upper gastrointestinal tract tumors. The last 2 phenotypes have low sensitivities, although they exhibit high specificities and PPVs (98.0\% and

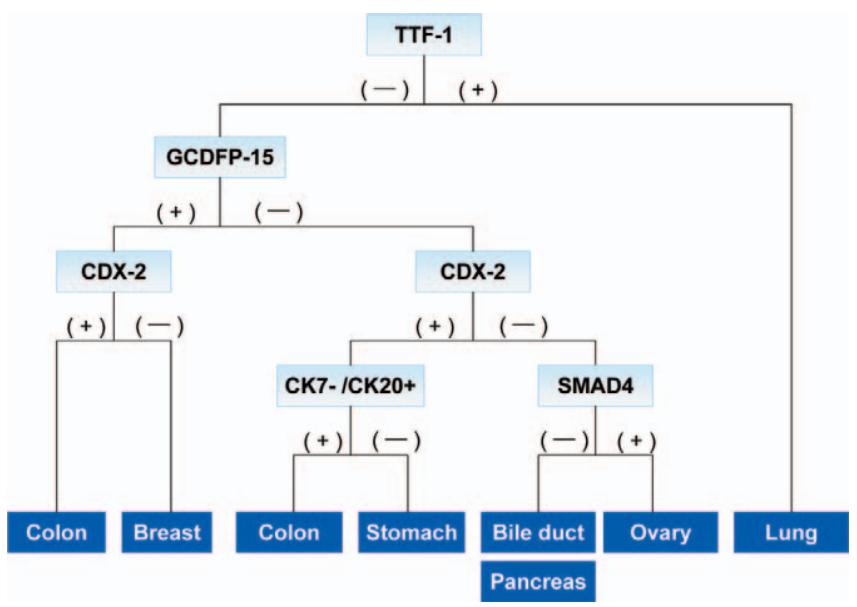

Figure 2. A decision tree was constructed based on the ranked specificities and sensitivities of 7 markers. Using this approach, the primary site was correctly predicted in $65.3 \%$ of cases. Pancreatic and bile duct origin tumors exhibited similar immunoprofiles. TTF-1 indicates thyroid transcription factor 1; GCDFP-15, gross cystic disease fluid protein 15; and $C K$, cytokeratin.

1564 Arch Pathol Lab Med—Vol 131, October 2007
$99.2 \%$, respectively, for specificity, and $87.1 \%$ and $85.7 \%$, respectively, for PPV).

\section{Testing of Combinatorial Immunostaining Profiles}

To validate the proposed combinatorial immunostaining profiles, we then extended the analysis to metastatic adenocarcinomas with known primary sites. We constructed one tissue microarray containing 60 metastatic adenocarcinomas from the seven main sites that was then immunostained with the 10 markers, scored, and the primary site predicted using the proposed combinatorial immunostaining profiles. The results for these predictions are presented in Table 4 . When this combination panel was applied to all metastatic cases, the correct primary site was determined in $75 \%$ of metastatic tumors. Six samples of breast origin were all classified correctly, and 14 of 15 metastatic adenocarcinomas of colorectal origin were correctly predicted. Eight cases (15.4\%) of metastatic adenocarcinomas, including 1 lung, 1 ovary, 3 stomach, and 3 pancreaticobiliary, were incorrectly predicted, which indicated that most of the misclassified tumors were metastases from a primary tumor in the stomach or pancreaticobiliary region. In 5 cases, the primary localization remained unknown (unclassifiable using our combined immunoprofiles).

On the other hand, in applying the decision tree to the same 60 metastatic adenocarcinomas, 33 cases (55\%) were classified, and 19 cases $(31.7 \%)$ were unclassified ( 8 cases [13.3\%] that failed to stain with any single marker were excluded). Moreover, the primary site was correctly predicted in only $24(46.2 \%)$ of 52 cases with the decision tree.

\section{COMMENT}

In the present study we evaluated a panel of $10 \mathrm{im}$ munohistochemical markers in 314 primary colorectal, gastric, pulmonary, pancreatic, breast, bile duct, and ovarian adenocarcinomas and 60 metastatic adenocarcinomas to develop immunohistochemical markers to predict the tissue origin of metastasis of unknown origin. For this purpose, 2 approaches were taken; namely, construction of a decision tree and design of combinatorial immunostaining profiles.

The sensitivities and specificities of 10 markers were determined for each tumor site. With the exception of TTF-1 and GCDFP-15, which are highly specific for lung and breast carcinomas, respectively, no single commercially 


\begin{tabular}{|c|c|c|c|c|c|c|c|c|c|c|c|}
\hline \multicolumn{3}{|c|}{ Pancreas } & \multicolumn{3}{|c|}{ Bile Duct } & \multicolumn{3}{|c|}{ Breast } & \multicolumn{3}{|c|}{ Ovary } \\
\hline $\begin{array}{c}\text { Sensitivity, } \\
\%\end{array}$ & $\begin{array}{c}\text { Specificity, } \\
\%\end{array}$ & $\begin{array}{c}\text { PPV, } \\
\%\end{array}$ & $\begin{array}{c}\text { Sensitivity, } \\
\%\end{array}$ & $\begin{array}{c}\text { Specificity, } \\
\%\end{array}$ & $\begin{array}{c}\text { PPV, } \\
\%\end{array}$ & $\begin{array}{c}\text { Sensitivity, } \\
\%\end{array}$ & $\begin{array}{c}\text { Specificity, } \\
\%\end{array}$ & $\begin{array}{c}\text { PPV, } \\
\%\end{array}$ & $\begin{array}{c}\text { Sensitivity, } \\
\%\end{array}$ & $\begin{array}{c}\text { Specificity, } \\
\%\end{array}$ & $\begin{array}{c}\text { PPV } \\
\%\end{array}$ \\
\hline 55.1 & 55.5 & 19.7 & 41.3 & 92.8 & 13.9 & 11.6 & 47.8 & 3.6 & 15.4 & 52.3 & 1.5 \\
\hline 0 & 82.2 & 0 & 0 & 82.4 & 0 & 0 & 82.6 & 0 & 0 & 71.4 & 0 \\
\hline 0 & 67.6 & 0 & 13 & 70.4 & 7.5 & 0 & 68.4 & 0 & 0 & 71.7 & 0 \\
\hline 8.2 & 69.2 & 5 & 26.1 & 72.8 & 15 & 4.6 & 69.2 & 2.5 & 30.8 & 73.1 & 5 \\
\hline 95.9 & 27.5 & 20.8 & 73.9 & 23.2 & 15 & 90.7 & 26.1 & 17.3 & 92.3 & 24.3 & 5.3 \\
\hline 0 & 86.2 & 0 & 4.3 & 87.2 & 5.9 & 2.3 & 86.9 & 2.9 & 0 & 87.9 & 0 \\
\hline 57.1 & 77.7 & 33.7 & 28.3 & 72 & 15.7 & 2.3 & 67.6 & 1.2 & 76.9 & 74.2 & 12 \\
\hline 20.4 & 47.4 & 7.1 & 13 & 46.4 & 4.3 & 67.4 & 56.1 & 20.7 & 76.9 & 54.1 & 7.1 \\
\hline 0 & 85 & 0 & 6.5 & 86.4 & 8.1 & 67.4 & 96.8 & 78.4 & 15.4 & 87.6 & 5.4 \\
\hline 0 & 92.7 & 0 & 0 & 92.8 & 0 & 34.9 & 98.8 & 83.3 & 0 & 93.6 & 0 \\
\hline
\end{tabular}

available marker has proved entirely site specific. GCDFP15 is generally considered a relatively specific and somewhat sensitive marker of breast cancers., ${ }^{3,14}$ Rates of GCDFP immunoreactivity in breast cancer have been reported to range from $43 \%$ to $77 \%$. $^{3,14-16}$ Possible reasons for the discrepancy between these rates and our data $(34.9 \%)$ might include differences in methods of immunohistochemistry, monoclonal antibody clones, tissue core size, and ethnic background of the patients. In any case, most data do not support high sensitivity of GCDFP-15 in breast cancer, thus limiting the practical utility of this marker.

Expression of CDX2 in tumors other than colorectal carcinoma has been previously reported. ${ }^{5}$ In this study, we observed CDX2 expression in $60.9 \%$ of stomach cancer cases and $13 \%$ of bile duct cancers. CDX2 expression alone does not reliably distinguish between colorectal adenocarcinomas and adenocarcinomas arising elsewhere in the gastrointestinal tract, particularly pancreaticobiliary and gastric adenocarcinomas, although the sensitivity of CDX2 for colorectal cancer is significantly higher $(93.9 \%)$ than for these other tumors.

SMAD4 is a tumor suppressor gene that is inactivated by allelic loss in $55 \%$ of pancreatic cancers, ${ }^{17}$ a finding that can be demonstrated by lack of immunostaining in these tumors. In several recent publications, SMAD4 was found to be positive in $100 \%$ of primary mucinous tumors of the ovary and in the majority $(89 \%)$ of colorectal carcinomas. ${ }^{18-21}$ Thus, negative staining for SMAD4 in adenocarcinoma is supportive of pancreatic tumor origin. Our study suggests that SMAD4 may be useful in distinguishing pancreaticobiliary from ovarian mucinous carcinomas, because up to $83.2 \%$ of pancreaticobiliary carcinomas lacked SMAD4 expression, whereas loss of SMAD4 expression was observed in only $23.1 \%$ of ovarian adenocarcinomas. However, SMAD4 is not helpful in the differential diagnosis of adenocarcinomas from the other organs (stomach, breast, lung, and ovary), because these tumors also expressed SMAD4 at high levels.

For the construction of a decision tree, some limitations should be recognized. No distinction was made between pancreatic and bile duct origin adenocarcinomas because of their similar immunoprofiles and low percentage of correct predictions. Dennis et $\mathrm{al}^{22}$ have proposed a classification scheme to enable better prediction of the primary site in patients with metastatic adenocarcinoma of unknown origin. In their study, they generated expression profiles for all 27 candidate markers in each of the seven (breast, colon, lung, ovary, pancreas, stomach, and prostate) main primary sites. From these data, statistical analysis led to a simplified diagnostic panel and decision tree containing 10 markers: CA 125, CDX2, CK7, CK20, ER, GCDFP-15, lysozyme, mesothelin, prostate-specific antigen, and TTF-1. Using this decision tree, the primary site was correctly predicted in $88 \%$ of cases. Interestingly, the 10 markers also were unreliable in determining the tissue origin of gastric and pancreaticobiliary cancers.

The advantage of using a combination panel of markers is improvement in the sensitivity and specificity of primary site detection. In this study, the majority of marker combinations had high specificity and sensitivity for diagnosing metastatic adenocarcinomas, especially those originating in breast, colon, and ovarian tissue. A combinatorial immunoprofile for pulmonary adenocarcinomas

\begin{tabular}{|c|c|c|c|c|}
\hline Organ & Phenotype & $\begin{array}{l}\text { Sensitivity, } \\
\quad \%\end{array}$ & $\begin{array}{l}\text { Specificity, } \\
\%\end{array}$ & $\begin{array}{c}\text { Positive } \\
\text { Predictive } \\
\text { Value, \% }\end{array}$ \\
\hline Colon & $\begin{array}{l}\text { TTF- } 1^{-} / \mathrm{CDX}^{+} / \mathrm{CK}^{-}-/ \mathrm{CK} 20^{+} \text {or } \\
\text { TTF- } 1^{-} / \mathrm{CDX}^{+} / \mathrm{CK}^{-}{ }^{-} / \mathrm{CK} 20^{-} /\left(\mathrm{CEA}^{+} \text {or } \mathrm{MUC}^{+}\right)\end{array}$ & 83.7 & 97.1 & 85.4 \\
\hline Ovary & 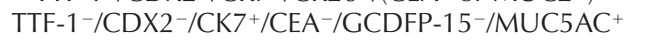 & 61.5 & 94.7 & 34.8 \\
\hline Breast & 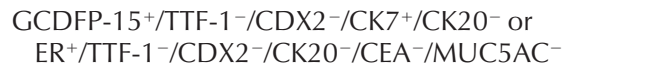 & 74.4 & 99.6 & 96.9 \\
\hline Lung & 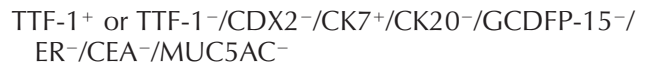 & 94 & 88.6 & 62.7 \\
\hline Pancreaticobiliary & TTF-1-/CDX2-/CK7+/CEA ${ }^{+} / \mathrm{MUC}^{-} \mathrm{AC}^{+}$ & 28.4 & 98 & 87.1 \\
\hline Stomach & TTF-1-/CDX2 ${ }^{+} / \mathrm{CK}^{-+} / \mathrm{CK} 20^{-}$ & 26 & 99.2 & 85.7 \\
\hline
\end{tabular}

* TTF-1 indicates thyroid transcription factor 1; CK, cytokeratin; CEA, carcinoembryonic antigen; GCDFP-15, gross cystic disease fluid protein 15; and ER, estrogen receptor. 


\begin{tabular}{|c|c|c|c|c|c|c|c|}
\hline \multirow[b]{2}{*}{ Actual Primary Site } & \multicolumn{7}{|c|}{ Predicted Primary Site } \\
\hline & Lung & Breast & Colon & Ovary & Stomach & Pancreaticobiliary & Missing \\
\hline Lung (12) & 10 & 1 & 0 & 0 & 0 & 0 & 1 \\
\hline Breast (6) & 0 & 6 & 0 & 0 & 0 & 0 & 0 \\
\hline Colon (15) & 0 & 0 & 14 & 0 & 0 & 0 & 1 \\
\hline Ovary (2) & 1 & 0 & 0 & 1 & 0 & 0 & 0 \\
\hline Stomach (8) & 1 & 0 & 0 & 1 & 3 & 1 & 2 \\
\hline Pancreaticobiliary (9) & 0 & 0 & 0 & 3 & 0 & 5 & 1 \\
\hline
\end{tabular}

also led to an increase in sensitivity over TTF- 1 alone. The major factor limiting the use of multiple-marker phenotypes is lack of sensitivity for several tumors. For example, the most predictive phenotype for pancreaticobiliary primary adenocarcinomas, TTF-1 $1^{-} / \mathrm{CDX} 2^{-} / \mathrm{CK}^{+} / \mathrm{CEA}^{+} /$ MUC5 $\mathrm{AC}^{+}$, was expressed in only $28.4 \%$ of cases; for gastric carcinomas, TTF- $1^{-} / \mathrm{CDX} 2^{+} / \mathrm{CK}^{+} / \mathrm{CK} 20^{-}$was expressed in only $26 \%$ of cases. Nevertheless, the final outcome of this approach showed a good overall result: $75 \%$ of tester samples (metastatic adenocarcinomas) could be correctly classified, with $15.4 \%$ classified incorrectly and $9.6 \%$ unclassified. The $25 \%$ of cases that were not correctly predicted by the immunoprofile algorithm, especially those of stomach or pancreaticobiliary origin, could be predicted by other additional immunomarkers. Recently, Mesothelin, MUC4, and CA 125 have been reported to be frequently expressed in pancreatic and bile duct adenocarcinomas. ${ }^{22-25}$ Use of these additional markers and clinical information could help improvement of prediction rates.

Many studies have defined the patterns of mucin expression in carcinomas of different organs. ${ }^{26-30}$ Previous studies have indicated that MUC2 and MUC5AC expressions were noted predominantly in colonic adenocarcinomas and mucinous ovarian adenocarcinomas, respectively. ${ }^{26}$ MUC5AC immunoreactivity was also observed in a higher percentage of pancreaticobiliary adenocarcinoma and a variable number of gastrointestinal tract tumors. ${ }^{26,29,30}$ In contrast, MUC2 was rarely expressed in adenocarcinomas of pancreaticobiliary origin,, 27 and many nongastrointestinal adenocarcinomas, such as adenocarcinomas of breast and lung, exhibited predominantly a MUC2- and MUC5AC-negative immunophenotype..$^{28}$ Lau et $\mathrm{a}^{28}$ demonstrated differential expression patterns of MUC1, MUC2, and MUC5AC in various carcinomas. The $\mathrm{MUC1}^{+} / \mathrm{MUC2}^{-}$/MUC5 $\mathrm{AC}^{-}$expression pattern was noted in breast cancer and the majority of pulmonary adenocarcinomas. The MUC1+/MUC2- $/ \mathrm{MUC}^{+} \mathrm{AC}^{+}$staining pattern was observed most often in adenocarcinomas of pancreas. However, use of these markers is likely to play a limited role in identification of the primary site in metastatic adenocarcinoma, because tumors from many tissue types exhibit overlapping patterns of MUC1, MUC2, and MUC5AC expression. In our study, a combination of additional immunomarkers allowed us to classify additional specific organs.

The male-female ratio of the patients with a primary adenocarcinoma of 5 organs excluding the breast and ovary was $2: 1$. We divided the tumors into male and female groups and compared the PPVs between both groups using combinatorial immunostaining profiles and found slight discrepancies. Many organs exhibited higher PPVs in the male group: $100 \%$ versus $0 \%$ in gastric cancers,
$56.1 \%$ versus $43.9 \%$ in colorectal cancers, $57.4 \%$ versus $42.6 \%$ in pulmonary cancers, and $42.1 \%$ versus $57.9 \%$ in pancreaticobiliary cancer patients. Furthermore, combinational immunostaining also showed a higher level of PPVs in metastatic adenocarcinomas from the male group than from the female group (61.8\% vs $34.3 \%)$. However, this result seems to lack reliability, because there were fewer female cases than male cases for each tissue type.

In summary, the designed combinations of immunostaining profiles are helpful in the diagnosis of tissue origin of metastatic adenocarcinomas and could offer fast and correct prediction of the primary site. The expression patterns of GCDFP-15, TTF-1, CDX2, CK7, CK20, MUC5AC, ER, and CEA are sufficient for classification in most cases, whereas expression of MUC2 and SMAD4 may help to classify some additional cases.

\section{References}

1. Pavlidis N, Briasoulis E, Hainsworth J, Greco FA. Diagnostic and therapeutic management of cancer of an unknown primary. Eur J Cancer. 2003;39:19902005.

2. Varadhachary GR, Abbruzzese JL, Lenzi R. Diagnostic strategies for unknown primary cancer. Cancer. 2004;100:1776-1785.

3. Brown RW, Campagna LB, Dunn JK, Cagle PT. Immunohistochemical identification of tumor markers in metastatic adenocarcinoma: a diagnostic adjunct in the determination of primary site. Am J Clin Pathol. 1997;107:12-19.

4. Hammar SP. Metastatic adenocarcinoma of unknown primary origin. Hum Pathol. 1998;29:1393-1402.

5. Bai YQ, Yamamoto H, Akiyama Y, et al. Ectopic expression of homeodomain protein CDX2 in intestinal metaplasia and carcinomas of the stomach. Cancer Lett. 2002;176:47-55.

6. Johansson L. Histopathologic classification of lung cancer: relevance of cytokeratin and TTF-1 immunophenotyping. Ann Diagn Pathol. 2004;8:259-267.

7. Levine PH, Joutovsky A, Cangiarella J, Yee H, Simsir A. CDX-2 expression in pulmonary fine-needle aspiration specimens: a useful adjunct for the diagnosis of metastatic colorectal adenocarcinoma. Diagn Cytopathol. 2006;34:191-195.

8. Moldvay J, Jackel M, Bogos K, et al. The role of TTF-1 in differentiating primary and metastatic lung adenocarcinomas. Pathol Oncol Res. 2004;10:8588.

9. Werling RW, Yaziji H, Bacchi CE, Gown AM. CDX2, a highly sensitive and specific marker of adenocarcinomas of intestinal origin: an immunohistochemical survey of 476 primary and metastatic carcinomas. Am J Surg Pathol. 2003;27: 303-310.

10. Yatabe Y, Mitsudomi T, Takahashi T. TTF-1 expression in pulmonary adenocarcinomas. Am J Surg Pathol. 2002;26:767-773.

11. Lagendijk JH, Mullink H, Van Diest PJ, Meijer GA, Meijer CJ. Tracing the origin of adenocarcinomas with unknown primary using immunohistochemistry: differential diagnosis between colonic and ovarian carcinomas as primary sites. Hum Pathol. 1998;29:491-497.

12. Rubin BP, Skarin AT, Pisick E, Rizk M, Salgia R. Use of cytokeratins 7 and 20 in determining the origin of metastatic carcinoma of unknown primary, with special emphasis on lung cancer. Eur J Cancer Prev. 2001;10:77-82.

13. Tot T. Cytokeratins 20 and 7 as biomarkers: usefulness in discriminating primary from metastatic adenocarcinoma. Eur J Cancer. 2002;38:758-763.

14. DeYoung BR, Wick MR. Immunohistologic evaluation of metastatic carcinomas of unknown origin: an algorithmic approach. Semin Diagn Pathol. 2000; 17:184-193.

15. Kaufmann O, Deidesheimer T, Muehlenberg M, Deicke P, Dietel M. Immunohistochemical differentiation of metastatic breast carcinomas from metastatic adenocarcinomas of other common primary sites. Histopathology. 1996; 29:233-240.

16. Tornos C, Soslow R, Chen S, et al. Expression of WT1, CA 125, and GCDFP-15 as useful markers in the differential diagnosis of primary ovarian car- 
cinomas versus metastatic breast cancer to the ovary. Am J Surg Pathol. 2005;29: 1482-1489.

17. Schutte M, Hruban RH, Hedrick L, et al. DPC4 gene in various tumor types. Cancer Res. 1996:56:2527-2530.

18. Baker PM, Oliva E. Immunohistochemistry as a tool in the differential diagnosis of ovarian tumors: an update. Int J Gynecol Pathol. 2005;24:39-55.

19. Ji H, Isacson C, Seidman JD, Kurman RJ, Ronnett BM. Cytokeratins 7 and 20, Dpc4, and MUC5AC in the distinction of metastatic mucinous carcinomas in the ovary from primary ovarian mucinous tumors: Dpc4 assists in identifying metastatic pancreatic carcinomas. Int J Gynecol Pathol. 2002;21:391-400.

20. Miyaki M, Kuroki T. Role of Smad4 (DPC4) inactivation in human cancer. Biochem Biophys Res Commun. 2003;306:799-804.

21. Wilentz RE, Su GH, Dai JL, et al. Immunohistochemical labeling for dpc4 mirrors genetic status in pancreatic adenocarcinomas: a new marker of DPC4 inactivation. Am J Pathol. 2000;156:37-43.

22. Dennis JL, Hvidsten TR, Wit EC, et al. Markers of adenocarcinoma characteristic of the site of origin: development of a diagnostic algorithm. Clin Cancer Res. 2005;11:3766-3772.

23. Hassan R, Laszik ZG, Lerner M, Raffeld M, Postier R, Brackett D. Mesothelin is overexpressed in pancreaticobiliary adenocarcinomas but not in normal pancreas and chronic pancreatitis. Am J Clin Pathol. 2005;124:838-845.
24. Jhala N, Jhala D, Vickers SM, et al. Biomarkers in diagnosis of pancreatic carcinoma in fine-needle aspirates. Am J Clin Pathol. 2006;126:572-579.

25. Cwik G, Wallner G, Skoczylas T, Ciechanski A, Zinkiewicz K. Cancer antigens 19-9 and 125 in the differential diagnosis of pancreatic mass lesions. Arch Surg. 2006;141:968-973; discussion 74.

26. Albarracin CT, Jafri J, Montag AG, Hart J, Kuan SF. Differential expression of MUC2 and MUC5AC mucin genes in primary ovarian and metastatic colonic carcinoma. Hum Pathol. 2000;31:672-677.

27. Chu PG, Schwarz RE, Lau SK, Yen Y, Weiss LM. Immunohistochemical staining in the diagnosis of pancreatobiliary and ampulla of Vater adenocarcinoma: application of CDX2, CK17, MUC1, and MUC2. Am J Surg Pathol. 2005; 29:359-367.

28. Lau SK, Weiss LM, Chu PG. Differential expression of MUC1, MUC2, and MUC5AC in carcinomas of various sites: an immunohistochemical study. Am J Clin Pathol. 2004;122:61-69.

29. Lee MJ, Lee HS, Kim WH, Choi Y, Yang M. Expression of mucins and cytokeratins in primary carcinomas of the digestive system. Mod Pathol. 2003; 16:403-410.

30. O'Connell FP, Wang HH, Odze RD. Utility of immunohistochemistry in distinguishing primary adenocarcinomas from metastatic breast carcinomas in the gastrointestinal tract. Arch Pathol Lab Med. 2005;129:338-347. 
Reproduced with permission of the copyright owner. Further reproduction prohibited without permission. 